\title{
Why Bayesian brains perform poorly on explicit probabilistic reasoning problems
}

\author{
Ryan Smith ${ }^{1}$, Maxwell J. D. Ramstead ${ }^{2,3}$, and Alex Kiefer ${ }^{3,4}$
}

Author affiliations:

${ }^{1}$ Laureate Institute for Brain Research, Tulsa, OK, USA.

${ }^{2}$ Wellcome Trust Centre for Human Neuroimaging, University College London, London, UK.

${ }^{3}$ VERSES Labs/Spatial Web Foundation, Los Angeles, California USA.

${ }^{4}$ Monash University, Melbourne, Victoria, AUS.

\section{Corresponding author:}

Ryan Smith

Laureate Institute for Brain Research

6655 S Yale Ave, Tulsa, OK 74136, USA

Email: rsmith@laureateinstitute.org

\section{Email Addresses for other authors:}

Maxwell J. D. Ramstead: maxwell.d.ramstead@gmail.com

Alex Kiefer: akiefer@gmail.com 


\begin{abstract}
There is a growing body of evidence suggesting that the neural processes underlying perception, learning, and decision-making approximate Bayesian inference. Yet, humans perform poorly when asked to solve explicit probabilistic reasoning problems. In response, some have argued that certain brain processes are Bayesian while others are not; others have argued that reasoning errors can be explained by either inaccurate generative models or limitations of approximation algorithms. In this paper, we offer a complementary perspective by considering how a Bayesian brain would implement conscious reasoning processes more generally. These considerations require making two distinctions, each of which highlights a fundamental reason why Bayesian brains should not be expected to perform well at explicit inference. The first distinction is between inferring probability distributions over hidden states and representing probabilities as hidden states. The former assumes that the brain's dynamics instantiate a form of approximate Bayesian inference, premised on a model of how observations are generated by hidden states of the world. In contrast, the latter assumes the brain represents probabilities themselves as hidden states namely, hypotheses about the correct answers to explicit reasoning problems. In this latter case, correctly inferring the most likely probability to report would implausibly require the brain to possess a generative model encoding Bayes' theorem itself. The second distinction is between inference and mental action. In addition to state inference, consciously solving Bayes' theorem requires the selection of a particular sequence of goal-directed cognitive actions (e.g., mental multiplication and addition, followed by division). While Bayesian brains infer probability distributions over action sequences, the possible sequences themselves often need to be learned. These considerations show that, regardless of the specific generative model in question or approximation algorithm employed, and even if all brain processes were Bayesian, an innate proficiency at solving explicit probabilistic reasoning problems should not be expected.
\end{abstract}

Keywords: Bayesian brain hypothesis; Bayes' theorem; Probabilistic inference; Decision biases; Reasoning; Active inference; Predictive processing 


\section{Introduction}

A substantial body of work has found support for the 'Bayesian brain hypothesis' - the broad idea that the brain represents uncertainty and approximates optimal probabilistic inference (Knill \& Pouget, 2004). According to this hypothesis, the brain (implicitly or explicitly) works with probabilities representing 1) prior beliefs ('priors') about the underlying states of the world, $p(s)$, and 2) the 'likelihood' that different observations will be generated by specific states, $p(o \mid s)$. It then uses these probabilities to infer updated 'posterior' beliefs, $p(s \mid o)$, about the state of the world after making new observations (i.e., after receiving new sensory input). This probabilistic belief updating (inference) process is based on Bayes' theorem:

$$
p(s \mid o)=\frac{p(o \mid s) p(s)}{p(o)}
$$

Here, $p(o)$ can be understood as the overall probability of making some observation across all possible states of the world included in a generative model (i.e., a model of how each possible state of the world generates observations) and can in principle be obtained by summing (or integrating) the term in the numerator across all values of $s$. To be clear, the claim behind the Bayesian brain hypothesis is not necessarily that the brain is literally engaging in Bayesian inference (although see (Kiefer, 2017) and (Hinton \& Sejnowski, 1983)). Rather, as we discuss below, it is sufficient that the brain's mechanics and dynamics can be interpreted as though they approximate this inference process.

In extant work on Bayesian perception and learning, considerable attention has been paid to 'predictive coding' models (Bastos et al., 2012; Bogacz, 2017; K. Friston, 2005; Rao \& Ballard, 1999), which propose that the brain embodies a generative model and continually updates its prior beliefs, $p(s)$, by minimizing 'prediction error' - the deviation between predicted and observed sensory input. That is, it continually identifies the set of beliefs in a model that best predict (and can therefore account for) the sensory data it receives. In these models, neural activity represents observations, states, and prediction errors, while the priors and likelihoods in the model are encoded within synaptic connection strengths that are adjusted more slowly over time based on statistical patterns across repeated observations (i.e., learning).

In research on decision-making and motor control, another prominent framework is 'active inference' - the idea that actions are chosen to minimize both uncertainty and the deviation between preferred and expected observations (e.g., see (Da Costa et al., 2020; K. J. Friston, Parr, \& de Vries, 2017; Smith, Friston, \& Whyte, 2022)). This is based on a model of the different observations generated by specific actions, $p(o \mid \pi)$, where each 'policy' $\pi$ corresponds to a possible action sequence. Using this model, a policy can be identified that maximizes the probability of desired outcomes (typically represented by a variable $C$ ). More specifically, a policy can be identified that minimizes the divergence between $p(o \mid \pi)$ and $p(o \mid C)$, which can be treated as a specific type of prediction error. The proposed neural implementation of active inference is similar to predictive coding in that neural activity represents probabilities over states and policies, while priors and likelihoods are represented within synaptic strengths that are learned more slowly through statistical associations between the neural activity representing policies, states, and observations. Active inference is also closely related to Bayesian decision theory, in which decisions are made to optimize the trade-off between the utility of each outcome and the probability of those outcomes under each possible action (Peterson, 2017). 
Outside of neuroscience, there are also several other lines of research in psychology and cognitive science modelling perception (Kersten, Mamassian, \& Yuille, 2004), categorization (Lake, Salakhutdinov, \& Tenenbaum, 2015; Sanborn, Griffiths, \& Navarro, 2010; Smith, Parr, \& Friston, 2019; Smith, Schwartenbeck, Parr, \& Friston, 2020), and several other cognitive processes in terms of Bayesian inference (Tenenbaum, Kemp, Griffiths, \& Goodman, 2011). Both empirical and simulation studies suggest that such models can account for a wide range of behavioral and neuroscientific findings (e.g., see (K. Friston, FitzGerald, Rigoli, Schwartenbeck, \& Pezzulo, 2017; Smith, Badcock, \& Friston, 2021; Smith, Mayeli, et al., 2021; Smith, Schwartenbeck, Stewart, et al., 2020; Walsh, McGovern, Clark, \& O'Connell, 2020; Whyte \& Smith, 2021; F. Xu \& J. Tenenbaum, 2007; F. Xu \& J. B. Tenenbaum, 2007)).

Given the prominence of this line of research, it is surprising to note a seemingly inconsistent set of results in the literature on judgment and decision-making. This separate body of work has documented a wide range of systematic reasoning biases in which humans appear not to conform to the prescriptions of probability theory (reviewed in (Kahneman, 2011)). This includes, for example, base rate neglect and the conjunction fallacy (Kahneman \& Tversky, 1973), the disjunction effect (Tversky \& Shafir, 1992), anchoring and adjustment effects (Epley \& Gilovich, 2001), probability matching (West \& Stanovich, 2003), and belief polarization (Mandelbaum, 2018), among others.

When asked to explicitly judge the probability of some hypothesis in light of new data and the relevant statistics, performance is also typically quite poor. For illustration, consider the following problem:

Approximately $1 \%$ of the population contracts disease $D$. The probability of showing a positive result $R_{p}$ on diagnostic test $T$ if one has disease $D$ is .8 (the probability of showing a negative result $R_{n}$ if one has disease $D$ is therefore .2 ). The probability of showing a positive result $R_{p}$ on test $T$ if one does not have the disease $(\sim D)$ is .3 (the probability of showing a negative result $R_{n}$ if one does not have the disease is therefore .7). Assume a person has a positive test result. What is the probability that they have disease $D$ ?

People tend to be very surprised to hear that the posterior probability of having disease $D$ given a positive test result, $p\left(D \mid R_{p}\right)$, is only around .007 - instead guessing it should be closer to .8. This is because people tend to underweight the prior probability of having the disease, $p(D)=.01$. They also tend not to fully incorporate the false positive rate; i.e., the likelihood of $p\left(R_{p} \mid \sim D\right)=$ .3 relative to $p\left(R_{p} \mid D\right)$. While the counterintuitive nature and difficulty of solving this problem is readily apparent experientially, it is unclear why we should perform poorly if our brains are Bayesian.

As discussed further below, there are several responses that have been offered to resolve the tension between the Bayesian brain hypothesis and the systematic reasoning biases documented within judgment and decision-making research (e.g., see (Chater et al., 2020; Williams, 2021)). Many people also do not consider our inability to solve explicit probabilistic inference problems to be a major threat to the Bayesian brain hypothesis. For example, one might hold that implicit processes outside of awareness are Bayesian, while explicit/voluntary cognitive processes are not (i.e., reminiscent of the distinction between 'system one' and 'system two' styles of cognition often discussed in contemporary psychology; see (Kahneman, 2011)). Or one might hold that 
verbally stated problems are just not in the format that the brain requires to perform inference (Oaksford \& Chater, 2007). Yet, it remains an interesting and under-explored question how we should understand explicit reasoning processes within a fully Bayesian brain and exactly why those processes should produce such poor intuitions and explicit responses when trying to solve problems such as the example of disease inference above.

In this paper we consider two fundamental reasons why we should not expect those with Bayesian brains to be good at solving this type of conscious probabilistic reasoning problem, even if all neural processes are Bayesian. The first reason is that representing probabilities of hypotheses is distinct from representing probabilities as hypotheses. While any form of statistical inference involves the former, the latter is also required to solve explicit, conscious reasoning problems such as the example above. Working with probabilities as hypotheses is more difficult when performing inference because it would require one's generative model to encode not only probabilistic relationships between states and observations, but also the relationships between probabilities themselves.

The second reason we should not expect Bayesian brains to excel at explicit probabilistic reasoning is that inference over states is distinct from policy selection. This is important because performing the mental calculations necessary to solve the type of probabilistic reasoning problem shown above requires executing a specific cognitive policy - and that policy needs to be learned.

Before presenting these arguments in detail, we first briefly review prior work on the discrepancy between the Bayesian and non-Bayesian aspects of our mental processes.

\section{Process distinctions and sampling}

Here we review prior work aimed at addressing the tension between the Bayesian brain hypothesis and studies showing seemingly irrational belief updating and decision-making. We do so primarily to differentiate this previous work from the target of explanation in the current paper. As will become clear below, this prior work has focused to a large extent on how a Bayesian brain might produce such errors due to particular process distinctions, prior beliefs, and/or constraints (among others). In contrast, our focus in subsequent sections will be on how to understand the way a Bayesian brain would engage in explicit reasoning and how this differs from other applications of its inferential machinery - in a manner that is independent of many of the processing vulnerabilities proposed to explain specific types of cognitive errors in previous work.

As mentioned above, one simple suggestion for resolving the dilemma posed by cases of irrational cognition is the possibility that some brain processes are Bayesian while others are not (Oaksford \& Chater, 2007). For example, perhaps domain-specific (modular) processes are Bayesian while domain-general cognitive processes are not; or perhaps perceptual and motor processes are Bayesian while abstract symbolic processes are not. While such distinctions are possible, it is not clear why these differences should be present. Further, when various confounds are taken into account, perceptual and cognitive processes show greater similarity (Jarvstad, Hahn, Rushton, \& Warren, 2013).

Another approach to solving this dilemma is to focus on approximation schemes that the brain may use to keep inference tractable. One suggestion along these lines has appealed to the idea that the brain may be a Bayesian sampler (Sanborn \& Chater, 2016). This is motivated by the 
fact that exact Bayesian inference is often not possible, and sampling approaches represent one major class of tractable approximation algorithms. While there are a number of different sampling approaches (many being variants of Markov Chain Monte Carlo [MCMC]; (Neal, 1993)), the basic idea is that, instead of inferring a full posterior probability distribution, one can simply start by picking a specific hypothesis and calculating its probability. Then one can stochastically move to another hypothesis and evaluate its probability relative to the first. This process is then iterated many times, while following particular rules that help ensure samples are taken from parts of the distribution with the highest probabilities. The more samples one takes, the better the approximation will be, converging toward the optimal Bayesian result as the number of samples approaches infinity.

Sampling methods have specific vulnerabilities that can provide potential explanations for observed errors in probabilistic reasoning. For example, the areas of the posterior distribution that are explored depend on the initial sampling location that is chosen. Further, sampling algorithms have difficulty comparing the probabilities of different events and, because the whole posterior cannot be explored, they can fail to identify distant locations with high probability (i.e., they can miss peaks separated by large, low-probability regions). These types of limitations have been argued to offer explanations for phenomena such as unpacking/conjunction effects (i.e., in which providing more detailed information leads events to be incorrectly judged as having higher probability (Rottenstreich \& Tversky, 1997; Sloman, Rottenstreich, Wisniewski, Hadjichristidis, \& Fox, 2004; Tversky \& Kahneman, 1983)) and base-rate neglect (i.e., in which the baseline frequency of an event is ignored; (Kahneman \& Tversky, 1973)), among others.

However, not all theories within the Bayesian brain framework appeal to sampling. For example, predictive coding and active inference models instead suggest the brain approximates Bayesian inference using variational free energy minimization (Bogacz, 2017; Smith et al., 2022). Although the mathematics are beyond the scope of this paper, the basic idea behind this approach is that (approximate) posterior beliefs are updated to minimize 'surprising' observations under a generative model. As touched on above, policies under this approach can also be selected to minimize uncertainty and maximize preferred observations in a similar fashion. As with sampling, although a brain engaging in free energy minimization will look as if it is performing Bayesian inference (i.e., its belief updating and policy selection will look approximately Bayes optimal on average and in the long run), it is not modeled as engaging in explicit Bayesian calculations. Instead, the system is simply mechanistically set up such that the dynamics minimize prediction error in a particular manner. Less work to date has explored whether this alternative approximation algorithm can also account for documented biases and reasoning errors (but see (Hohwy, 2017)).

One further suggestion for addressing certain cases of seemingly non-Bayesian cognition is appeal to Bayesian decision theory (Peterson, 2017), in which Bayes-optimal cognition further requires consideration of expected utility. For example, one common finding is that humans seem to update beliefs in a Bayesian manner if irrelevant to the self, but do not do so when the evidence presented is inconsistent with preferred beliefs (e.g., see (Eil \& Rao, 2011)). However, if the brain conforms to Bayesian decision theory (or active inference), it has been suggested that these types of 'motivated cognition' can be accounted for (e.g., see (Williams, 2021)). One specific way this could arise is if utility is assigned to holding some beliefs over others. Bayes- 
optimal decision making could then maximize utility by selecting cognitive actions (e.g., selective attention) that maintain preferred beliefs. Another example of appeals to Bayesian decision theory involves the expected costs of some mistakes vs. others. There are well-known examples where false positives (e.g., mistaking a stick for a snake) are much less costly than false negatives (e.g., mistaking a snake for a stick). In such cases, one could argue that biased belief updating (e.g., favoring snakes over sticks) is non-Bayesian. Yet, similar to the cases of motivated cognition mentioned above, this type of belief updating (and subsequent action selection) can still be modelled as Bayes-optimal once the utility of different outcomes is factored in (e.g., the strong negative utility of a snake). One could also argue that evolution selected for brains with precise prior beliefs favoring stimuli that would be costly to misperceive (e.g., a strong prior favoring snakes), such that false positives are Bayes-optimal.

To summarize: while plausible, the theoretical strategies proposed to date for reconciling findings suggesting brains do vs. do not approximate Bayesian inference depend on specific assumptions that may or may not hold (e.g., specific process distinctions, priors, utility functions, or types of approximation algorithms). To complement this work, it could therefore be useful to explore broader computational considerations that do not depend on these specific assumptions. Further, most of the work described above does not address problems requiring application of Bayes' theorem itself (i.e., many seek to explain errors that also draw on implicit inference processes). It may therefore also be useful to more specifically address the way we should understand processes associated with explicit Bayesian reasoning in a Bayesian brain. That is, if conscious reasoning is underpinned by Bayesian processes, why do those processes lead to poor performance with explicit probabilistic inference?

\section{Why Bayesian brains should not be optimal mathematical reasoners}

In this section, we describe another complementary perspective on why Bayesian brains should not be expected to perform optimally on probabilistic reasoning problems, with a focus on how a Bayesian architecture could be engaged to solve such problems. Unlike the work described above, our argument does not depend on any specific approximation strategy or process distinction. It only appeals to the abstract requirements of probabilistic reasoning problems at a computational level of description and how they differ from other problems that can be solved effectively by a Bayesian brain.

\section{Inferring probabilities over states vs. as states}

The first component of our argument appeals to an important distinction between the process of inference and the content being inferred. In Bayesian perception, for example, the brain infers the posterior probability of different possible hidden causes of its sensations; e.g., inferring the probability of a box vs. a ball conditioned on a pattern of visual input. Here, 'box' and 'ball' are the contents being inferred, and an individual will report on those contents (i.e., they will report seeing one vs. the other, corresponding to the content with the highest probability). In contrast, consider again the problem of inferring the posterior probability of disease $D$ in the opening section. In this case, the individual is being asked to report the probability itself. In other words, the probability is the content being inferred. Thus, while in perception the brain is inferring probabilities over hidden states, explicit probabilistic reasoning problems require an individual to make inferences about probabilities treated as hidden states (see Figure 1). 


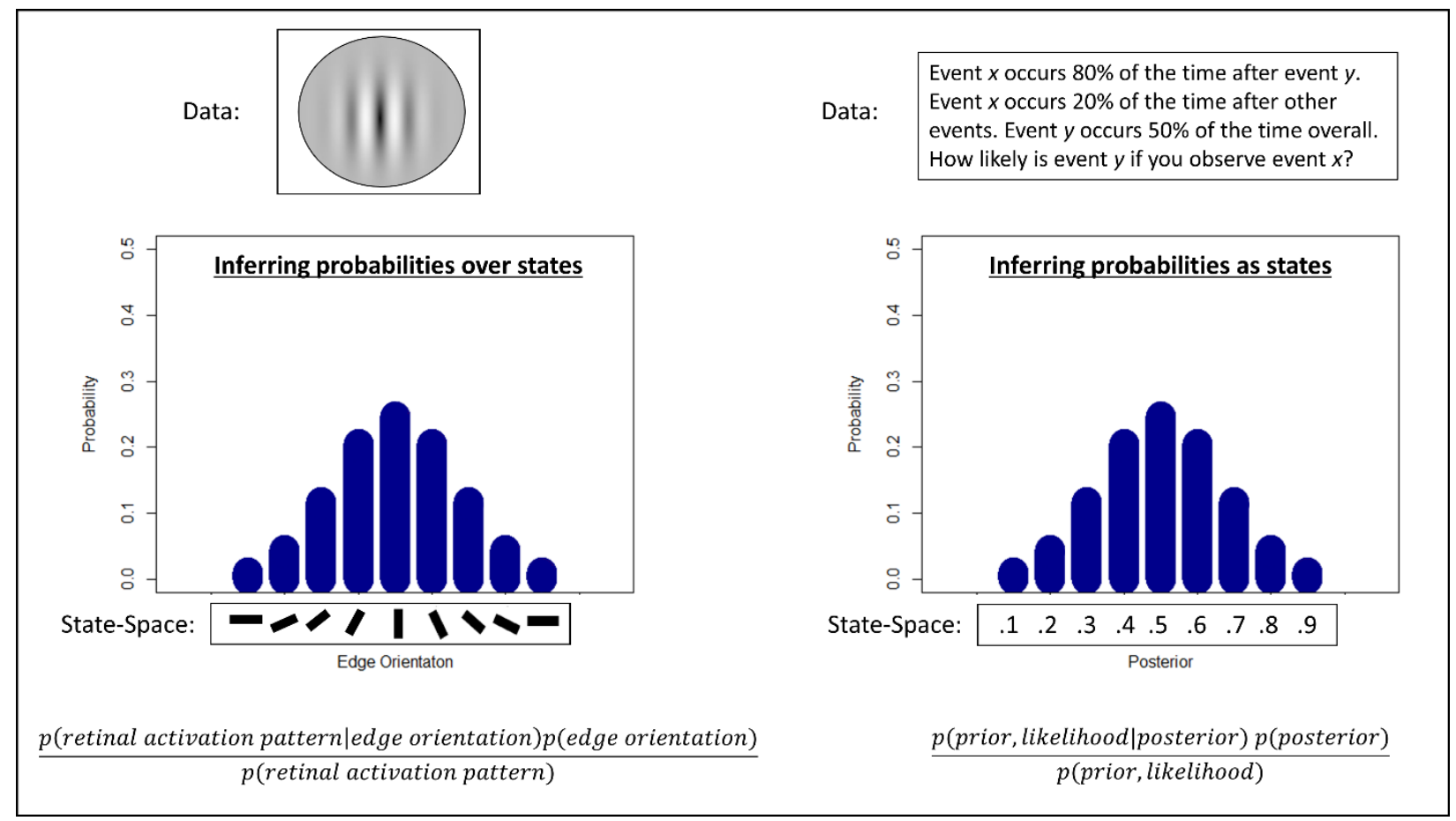

Figure 1. Left: Probabilistic inference over states. In this example, the sensory data is the retinal activation pattern elicited by the vertical grating stimulus at the top. The state-space includes possible edge orientations. Here, neural activity may represent the posterior probability of each edge orientation by approximating the solution to Bayes' theorem shown at the bottom. Right: Inferring probabilities as states. In this case, the data in an explicit reasoning problem is the stated prior probability and likelihood associated with two events. The state-space includes the possible answers (posterior probabilities) to the problem. The solution to the problem requires inferring the most likely posterior probability based on the prior and likelihood, as shown at the bottom. The ability to perform inference in this latter case is not entailed by the Bayesian brain hypothesis, as there is no reason to expect a Bayesian brain should possess a generative model treating probabilities as states and observations.

Thus, consider how an in silico agent would be set up to solve this problem. Unlike in perception, the 'data' used for inference are not the types of 'raw' sensory input processed within sensory cortices, but instead the probabilities (priors and likelihoods) stated within the problem description. That is, the 'data' used for inference include, in this case, $p(D)=.01, p\left(R_{p} \mid D\right)=.8$, and $p\left(R_{p} \mid \sim D\right)=.3$. A Bayesian agent would then have to use Bayes' theorem to infer the most likely posterior, $p\left(D \mid R_{p}\right)$. Crucially, to infer the most likely value for $p\left(D \mid R_{p}\right)$ would require the agent to have a model of Bayes' theorem itself. That is, it would need to know $p$ (prior, likelihood|posterior) and it would also possess a prior belief about the most likely posterior, $p$ (posterior). For example, after solving several similar word problems, it might come to believe the posterior is usually between .5 and .8 .

The overall point here is that applying the machinery within a Bayesian brain to engage in explicit probabilistic reasoning entails the use of a generative model of probabilities - that is, a generative model specifying the relationship between numbers labeled as priors, likelihoods, and posteriors. Because there is no reason to expect that a Bayesian brain should possess such a model, the ability to solve explicit reasoning problems optimally should not be expected. Instead, Bayesian brains are only expected to possess models of the causes of their sensations and to use such models to infer the most likely causes given those sensations.

\section{Inference vs. mental action}


The second component of our argument appeals to the notion of mental action. As described above, computational models of decision processes, such as active inference (as well as reinforcement learning; (Sutton \& Barto, 1998)), require agents to either learn or infer the optimal sequence of actions (i.e., the optimal policy) to accomplish some objective (e.g., maximize desired outcomes, minimize uncertainty). Importantly, not all action sequences must include overtly observable behavior. Some action sequences can be purely mental (i.e., cognitive policies), such as choosing a covert target of selective attention or choosing what information to maintain/manipulate in working memory (Metzinger, 2017); for examples of computational models simulating this kind of cognitive policy selection, see (Sandved-Smith et al., 2021; Smith, Lane, Parr, \& Friston, 2019; Smith, Parr, et al., 2019). Here we highlight the fact that solving probabilistic reasoning problems also appears to require learning and executing cognitive policies, corresponding to the phenomenology of engaging in voluntary, goal-directed mental action in such cases. We show how this may also be a main point of failure underlying the poor probabilistic reasoning within Bayesian brains.

Consider an undergraduate student seeking a degree in mathematics. When taking a course on probability theory, they will be taught Bayes' theorem and asked to use it to solve for posterior probabilities. The process of gaining proficiency in solving such problems, especially without a pencil and paper, requires learning a sequence of mental actions. For example, in the problem of inferring $p\left(D \mid R_{p}\right)$ presented above, one would first choose to hold the values of $p(D)$ and $p\left(R_{p} \mid D\right)$ in working memory. Following Bayes' theorem, one would then manipulate these values in working memory to mentally multiply them and find the joint probability $p\left(R_{p}, D\right)$. One would then mentally add together $p\left(R_{p} \mid D\right)$ and $p\left(R_{p} \mid \sim D\right)$ to obtain $p\left(R_{p}\right)$. Finally, one would divide $p\left(R_{p}, D\right)$ by $p\left(R_{p}\right)$ to find $p\left(D \mid R_{p}\right)$.

The important point here is that, when the act of solving Bayes' theorem is cast as a cognitive policy (i.e., a series of mental actions), it is clear why a Bayesian brain should not be expected to come pre-equipped with that policy. Instead, such policies are learned, and a Bayesian brain will only be innately equipped with the ability to infer which of its learned policies are most likely to accomplish its objective under a model (i.e., to infer a distribution over policies; see Figure 2). This matches well with the phenomenological experience of learning to calculate solutions to Bayesian inference problems, becoming proficient with practice, and then selecting that policy whenever it is expected to yield a desired outcome (e.g., passing a course). 


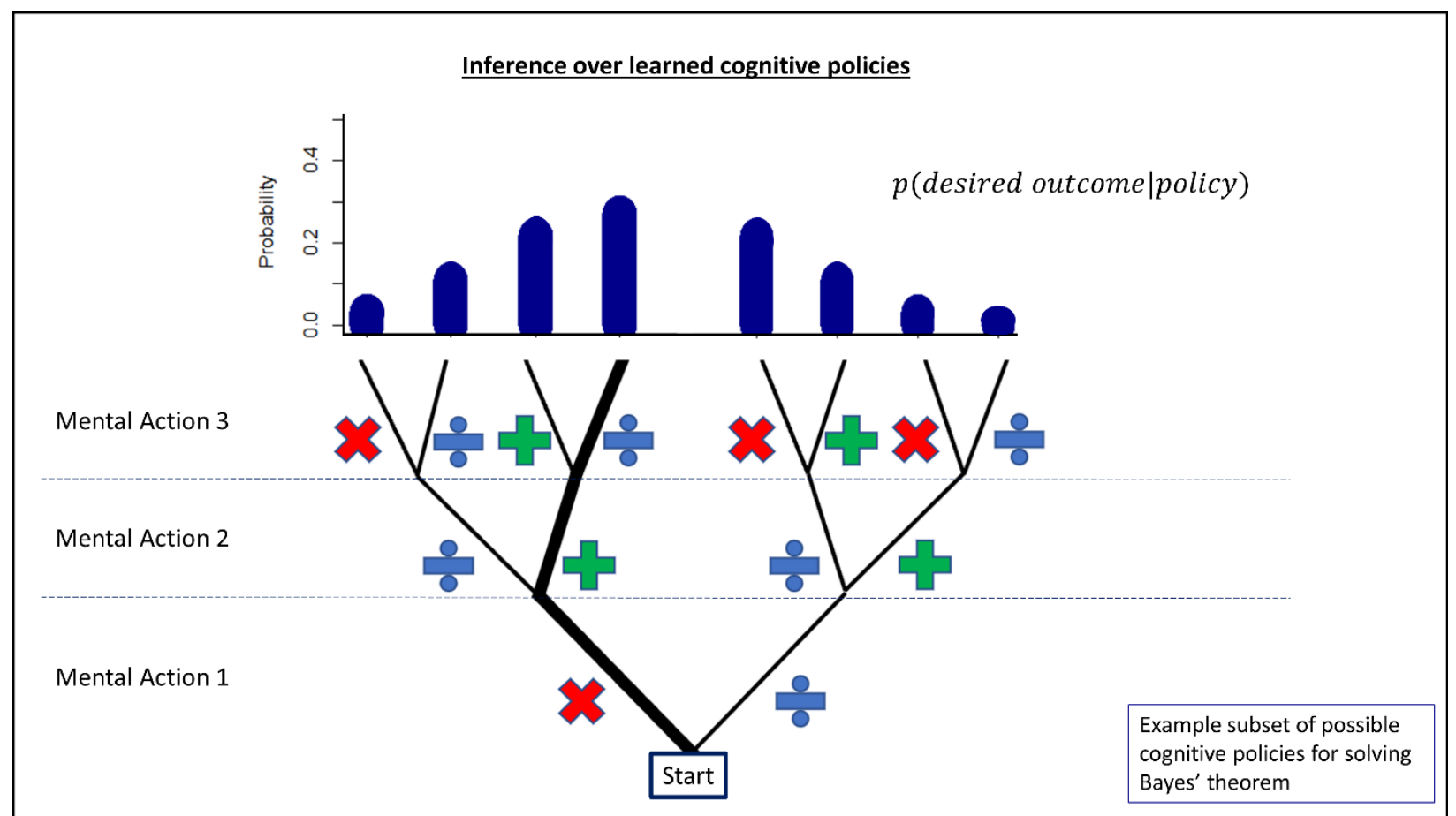

Figure 2. Probabilistic inference over policies. The decision tree at the bottom illustrates an example subset of possible cognitive policies for mental calculation (other possible calculation sequence are omitted for clarity). When asked to solve a probabilistic reasoning problem, the brain would need to infer the probability that each policy will generate the desired outcome of stating the correct answer. The sequence of thick lines indicates the correct sequence to solve Bayes' theorem (i.e., multiply the prior and likelihood to get the joint probability, sum the likelihood under all states to get the overall 'marginal' probability of the desired outcome, and then divide the joint probability by the marginal probability). While a Bayesian brain infers probabilities over policies, to do so correctly in this case will require having learned the 'Bayes' theorem policy' indicated by the thick lines. Without mathematical training, there is no reason to expect a Bayesian brain to innately possess knowledge of this policy.

\section{Discussion}

In this short paper, we have highlighted two reasons why Bayesian brains should not be expected to perform well on explicit probabilistic reasoning problems. The first is that directly inferring the posterior probability of some event based on the data provided in a probabilistic reasoning problem would implausibly require the brain to possess an explicit model of the relationships between types of probability distributions specified by Bayes' theorem (i.e., treating those probabilities as states and observations). As reviewed above, a Bayesian brain does not require explicit representation of these relationships. The second is that step-by-step calculation of Bayes' theorem requires the possession of a particular cognitive policy that could only plausibly be learned through instruction. Both of these processes are distinct from the central tenet of the Bayesian brain theory, which is that brains perform probabilistic inference over hidden states and policies. That is, they infer the probability of things, and not probabilities themselves.

One interesting consideration is that our account of explicit inference and cognitive action could also apply to the way Bayesian brains implement conscious, goal-directed thinking/reasoning more generally; hence, our arguments could also apply to human performance in other reasoning domains, such as deductive inference. For example, as in the case of Bayesian reasoning, humans show innate difficulties 
in their capacity to judge the validity of deductive arguments - such as, being prone to systematic errors like affirming the consequent (Wason, 1971). However, as with Bayesian reasoning, humans can also improve with training (e.g., within courses in formal logic), suggesting a process of learning new stateoutcome mappings and mental action sequences. Our account would also predict (as appears true anecdotally) that skills in simple deductive reasoning (e.g., determining validity) should be easier to acquire than Bayesian reasoning, since they require learning fewer mental actions and involve inference over a smaller number of states (e.g., a binary decision about implication rather than the computation of one numerical value given others).

A possible objection is that solving explicit probabilistic reasoning problems need not require representing probabilities as states and observations (i.e., which would be associated with changes in neural activity over fast timescales). Instead, one might claim that the brain encodes the stated priors and likelihoods the same way it does in perception. Under this possible alternative, the act of reading the information would lead individuals to imagine a concrete scenario (at a perceptual level) and then rapidly reconfigure the strength of synaptic connections encoding the priors and likelihoods associated with the imagined states and observations. If this were the case, a Bayesian brain might still be expected to show approximately optimal inference. Although we cannot fully rule out this alternative, it appears implausible for multiple reasons. First, this rapid 're-programming' would require additional mechanisms not postulated by Bayesian brain theories. Second, the statistical learning mechanisms leading to changes in synaptic strengths appear to operate too slowly, based on patterns across repeated observations that are not present in these types of reasoning problems. Third, there is no evidence suggesting individuals reliably imagine concrete examples when presented with abstract probabilities in these problems (in contrast, this may be more likely when the same problems are presented in terms of frequencies, in which performance improves - perhaps because frequencies are easier to imagine and more similar to the sequential information format processed in perception (Gigerenzer \& Hoffrage, 1995)).

It is also important to clarify that the points made here should not be seen as in competition with prior work showing how specific reasoning errors can be explained by the vulnerabilities of approximation algorithms (Chater et al., 2020; Sanborn \& Chater, 2016), or with other suggestions about how cognitive reasoning processes may differ in character from perceptual and/or other domain-specific processes (Oaksford \& Chater, 2007). Our point is simply that, independent of any approximation algorithm or potential process distinction, a Bayesian brain need not be set up to solve such problems (i.e., as they require models/policies on which inference is performed, as opposed to the ability to perform inference using models). This is fully consistent with the idea that specific reasoning errors are explained by the need to use approximation algorithms, as our explanation just applies to Bayesian reasoning generally. Our argument about the need for cognitive policies could also be seen as offering an explanation for differences between perception and one particular type of cognition (i.e., mental action selection). Namely, the type of cognition relevant for explicit Bayesian inference is characterized by the selection of a learned sequence of mental actions, whereas Bayesian perception is a process of inference over states. This is important specifically because it addresses the issue of how fully Bayesian machinery can be used in conscious, voluntary reasoning - which has not been the focus of previous proposals. It also highlights specifically why this leads such machinery to perform poorly. Namely, despite the inference process itself remaining approximately Bayesian, the required likelihood function and policy space needed for Bayesian machinery to solve these problems are: 1) not plausible candidates for being evolutionarily programmed in, and 2) very complex and difficult to learn.

We conclude that explicit probabilistic reasoning errors, no matter how egregious, need not be seen as a threat to the Bayesian brain hypothesis. In fact, while the ability to approximate Bayesian inference offers 
clear fitness advantages, very unlikely evolutionary pressures would have been necessary to select for brains that come pre-equipped with a model of Bayes' theorem or an innate mental policy for solving it. These considerations complement work aimed at explaining the reasons for specific reasoning errors, but highlight why poor performance on explicit inference problems need not rest on a priori assumptions about psychological process distinctions or the specific approximation strategies a Bayesian brain might use. Finally, even if poor performance on explicit reasoning problems is not viewed as a threat to the Bayesian brain hypothesis, the considerations presented here provide a detailed articulation of why this is the case - even if all brain processes were Bayesian.

Acknowledgment: The authors would like to thank Jakob Hohwy for reading and providing feedback on the manuscript.

\section{References}

Bastos, A. M., Usrey, W. M., Adams, R. A., Mangun, G. R., Fries, P., \& Friston, K. J. (2012). Canonical microcircuits for predictive coding. Neuron, 76(4), 695-711. doi:10.1016/j.neuron.2012.10.038

Bogacz, R. (2017). A tutorial on the free-energy framework for modelling perception and learning. $J$ Math Psychol, 76(Pt B), 198-211. doi:10.1016/j.jmp.2015.11.003

Chater, N., Zhu, J. Q., Spicer, J., Sundh, J., León-Villagrá, P., \& Sanborn, A. (2020). Probabilistic biases meet the Bayesian brain. Current Directions in Psychological Science, 29(5), 506-512.

Da Costa, L., Parr, T., Sajid, N., Veselic, S., Neacsu, V., \& Friston, K. (2020). Active inference on discrete state-spaces: A synthesis. J Math Psychol, 99, 102447. doi:10.1016/j.jmp.2020.102447

Eil, D., \& Rao, J. M. (2011). The Good News-Bad News Effect: Asymmetric Processing of Objective Information about Yourself. American Economic Journal: Microeconomics, 3, 114-138.

Epley, N., \& Gilovich, T. (2001). Putting adjustment back in the anchoring and adjustment heuristic: Differential processing of self-generated and experimenter-provided anchors. Psychological Science, 12(5), 391-396. doi:https://doi.org/10.1111/1467-9280.00372

Friston, K. (2005). A theory of cortical responses. Philos Trans R Soc Lond B Biol Sci, 360(1456), 815-836. doi:10.1098/rstb.2005.1622

Friston, K., FitzGerald, T., Rigoli, F., Schwartenbeck, P., \& Pezzulo, G. (2017). Active Inference: A Process Theory. Neural Comput, 29(1), 1-49. doi:10.1162/NECO_a_00912

Friston, K. J., Parr, T., \& de Vries, B. (2017). The graphical brain: Belief propagation and active inference. Netw Neurosci, 1(4), 381-414. doi:10.1162/NETN_a_00018

Gigerenzer, G., \& Hoffrage, U. (1995). How to improve Bayesian reasoning without instruction: Frequency formats. Psychological review, 102(4), 684-704.

Hinton, G. E., \& Sejnowski, T. J. (1983). Optimal perceptual inference. Proceedings of the IEEE conference on Computer Vision and Pattern Recognition, 448.

Hohwy, J. (2017). Priors in Perception: Top-Down Modulation, Bayesian Perceptual Learning Rate, and Prediction Error Minimization. Consciousness and Cognition, 47, 75-85.

Jarvstad, A., Hahn, U., Rushton, S. K., \& Warren, P. A. (2013). Perceptuo-motor, cognitive, and description-based decision-making seem equally good. Proc Natl Acad Sci U S A, 110(40), 1627116276. doi:10.1073/pnas.1300239110

Kahneman, D. (2011). Thinking, Fast and Slow.

Kahneman, D., \& Tversky, A. (1973). On the psychology of prediction. Psychological review, 80(4), 237251. 
Kersten, D., Mamassian, P., \& Yuille, A. (2004). Object perception as Bayesian inference. Annu Rev Psychol, 55, 271-304. doi:10.1146/annurev.psych.55.090902.142005

Kiefer, A. (2017). Literal perceptual inference. In T. Metzinger \& W. Wiese (Eds.), Philosophy and predictive processing. Frankfurt, Germany.

Knill, D. C., \& Pouget, A. (2004). The Bayesian brain: the role of uncertainty in neural coding and computation. Trends Neurosci, 27(12), 712-719. doi:10.1016/j.tins.2004.10.007

Lake, B. M., Salakhutdinov, R., \& Tenenbaum, J. B. (2015). Human-level concept learning through probabilistic program induction. Science, 350(6266), 1332-1338. doi:10.1126/science.aab3050

Mandelbaum, E. (2018). Troubles with Bayesianism: An introduction to the psychological immune system. Mind \& Language, 1-17. doi:https://doi.org/10.1111/mila.12205

The Problem of Mental Action (2017).

Neal, R. M. (1993). Probabilistic inference using Markov chain Monte Carlo methods. Toronto, ON, Canada: Department of Computer Science, University of Toronto.

Oaksford, M., \& Chater, N. (2007). Bayesian rationality: The probabilistic approach to human reasoning: Oxford University Press.

Peterson, M. (2017). An Introduction to Decision Theory. Cambridge: Cambridge University Press.

Rao, R. P., \& Ballard, D. H. (1999). Predictive coding in the visual cortex: a functional interpretation of some extra-classical receptive-field effects. Nat Neurosci, 2(1), 79-87. doi:10.1038/4580

Rottenstreich, Y., \& Tversky, A. (1997). Unpacking, repacking, and anchoring: advances in support theory. Psychol Rev, 104(2), 406-415. doi:10.1037/0033-295x.104.2.406

Sanborn, A. N., \& Chater, N. (2016). Bayesian Brains without Probabilities. Trends Cogn Sci, 20(12), 883893. doi:10.1016/j.tics.2016.10.003

Sanborn, A. N., Griffiths, T. L., \& Navarro, D. J. (2010). Rational approximations to rational models: Alternative algorithms for category learning. Psychological review, 117, 1144-1167.

Sandved-Smith, L., Hesp, C., Mattout, J., Friston, K., Lutz, A., \& Ramstead, M. J. D. (2021). Towards a computational phenomenology of mental action: modelling meta-awareness and attentional control with deep parametric active inference. Neurosci Conscious, 2021(2), niab018. doi:10.1093/nc/niab018

Sloman, S., Rottenstreich, Y., Wisniewski, E., Hadjichristidis, C., \& Fox, C. R. (2004). Typical versus atypical unpacking and superadditive probability judgment. J Exp Psychol Learn Mem Cogn, 30(3), 573-582. doi:10.1037/0278-7393.30.3.573

Smith, R., Badcock, P., \& Friston, K. J. (2021). Recent advances in the application of predictive coding and active inference models within clinical neuroscience. Psychiatry Clin Neurosci, 75(1), 3-13. doi:10.1111/pcn.13138

Smith, R., Friston, K., \& Whyte, C. (2022). A Step-by-Step Tutorial on Active Inference and its Application to Empirical Data. Journal of Mathematical Psychology, (In Press).

Smith, R., Lane, R. D., Parr, T., \& Friston, K. J. (2019). Neurocomputational mechanisms underlying emotional awareness: Insights afforded by deep active inference and their potential clinical relevance. Neuroscience \& Biobehavioral Reviews, 107, 473-491. doi:10.1016/j.neubiorev.2019.09.002

Smith, R., Mayeli, A., Taylor, S., Al Zoubi, O., Naegele, J., \& Khalsa, S. S. (2021). Gut inference: A computational modelling approach. Biological psychology, 164, 108152. doi:10.1016/j.biopsycho.2021.108152

Smith, R., Parr, T., \& Friston, K. J. (2019). Simulating Emotions: An Active Inference Model of Emotional State Inference and Emotion Concept Learning. Frontiers in psychology, 10, 2844. doi:10.3389/fpsyg.2019.02844 
Smith, R., Schwartenbeck, P., Parr, T., \& Friston, K. J. (2020). An Active Inference Approach to Modeling Structure Learning: Concept Learning as an Example Case. Front Comput Neurosci, 14, 41. doi:10.3389/fncom.2020.00041

Smith, R., Schwartenbeck, P., Stewart, J. L., Kuplicki, R., Ekhtiari, H., Investigators, T., \& Paulus, M. P. (2020). Imprecise Action Selection in Substance Use Disorder: Evidence for Active Learning Impairments When Solving the Explore-exploit Dilemma. Drug and Alcohol Dependence, 215, 108208.

Sutton, R., \& Barto, A. (1998). Reinforcement Learning: An Introduction.

Tenenbaum, J. B., Kemp, C., Griffiths, T. L., \& Goodman, N. D. (2011). How to grow a mind: statistics, structure, and abstraction. Science, 331(6022), 1279-1285. doi:10.1126/science.1192788

Tversky, A., \& Kahneman, D. (1983). Extensional versus intuitive reasoning: The conjunction fallacy in probability judgment. Psychological review, 90(4), 293.

Tversky, A., \& Shafir, E. (1992). The disjunction effect in choice under uncertainty. Psychological Science, 3(5), 305-309. doi:https://doi.org/10.1111/j.1467-9280.1992.tb00678.x

Walsh, K. S., McGovern, D. P., Clark, A., \& O'Connell, R. G. (2020). Evaluating the neurophysiological evidence for predictive processing as a model of perception. Ann N Y Acad Sci, 1464(1), 242-268. doi:10.1111/nyas.14321

Wason, P. C. (1971). Problem solving and reasoning. British Medical Bulletin, 27(3), 206-210. doi:https://doi.org/10.1093/oxfordjournals.bmb.a070854

West, R. F., \& Stanovich, K. E. (2003). Is probability matching smart? Associations between probabilistic choices and cognitive ability. Memory \& Cognition, 31(2), 243-251. doi:https://doi.org/10.3758/BF03194383

Whyte, C., \& Smith, R. (2021). The predictive global neuronal workspace: A formal active inference model of visual consciousness. Prog Neurobiol, 199, 101918. doi:10.1016/j.pneurobio.2020.101918

Williams, D. (2021). Epistemic Irrationality in the Bayesian Brain. The British Journal for the Philosophy of Science, 72(4). doi:https://doi.org/10.1093/bjps/axz044

Xu, F., \& Tenenbaum, J. (2007). Word Learning as Bayesian Inference. Psychological review, 114, 245272.

Xu, F., \& Tenenbaum, J. B. (2007). Sensitivity to sampling in Bayesian word learning. Dev Sci, 10(3), 288297. doi:10.1111/j.1467-7687.2007.00590.x 